\title{
Failure Analysis of a Launch Vehicle Umbilical Shutter Mechanism During Vibration Qualification
}

\author{
V. Murugesan • P. S. Sreejith • M. Gopakumar • P. Damodaran • \\ M. Premdas
}

Submitted: 20 November 2017/Published online: 9 March 2018

(c) ASM International 2018

\begin{abstract}
Payload fairing (PLF) of a launch vehicle is exposed to harsh vibration environments due to jet noise during liftoff and in-flight aerodynamic noise. Accordingly, the systems mounted on the payload fairing are to be qualified for the vibration levels, predicted corresponding to the envelope of acoustic spectrums at critical instants of atmospheric flight. This paper presents a detailed study of a failure observed on the payload cooling umbilical system, mounted on the cylindrical portion of the PLF structure, during its design qualification vibration testing. The umbilical shutter inadvertently opened during the test. The vibration responses on the shutter, the dynamic behavior of the system, and the forces and moments on the mechanism are analyzed, and the physics of failure is understood. The design marginality is identified, and the shutter locking mechanism reconfigured to achieve the desired level of robustness in the system.
\end{abstract}

Keywords Failure mode - Umbilical shutter .

Vibration response $\cdot$ Transmissibility $\cdot$ Forces and moments

\section{Abbreviations}

FE Finite element

OASPL Overall acoustic sound pressure level

PLF Payload fairing

PSD Power spectral density

V. Murugesan $(\bowtie) \cdot$ M. Gopakumar · P. Damodaran ·

M. Premdas

Vikram Sarabhai Space Centre, Thiruvananthapuram 695022,

India

e-mail: v_murugesan@vssc.gov.in

P. S. Sreejith

Cochin University of Science and Technology, Cochin 682022, India

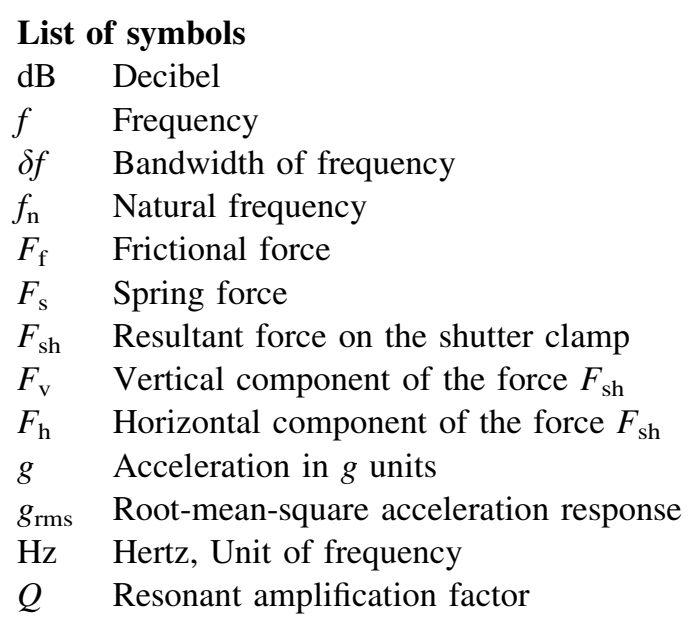

\section{Introduction}

The spacecraft is assembled over the upper stage of a launch vehicle, through an interface structure called payload adapter. During the atmospheric ascent phase, the spacecraft is protected from the aerodynamic loads and heating by a payload fairing (PLF). The fairing is separated, when the aerothermal heat flux on the payload is less than $1135 \mathrm{~W} / \mathrm{cm}^{2}$ [1]. During the ground operations till liftoff, the spacecraft and the vehicle systems are tested for their functional performance. During this phase, the vehicle and spacecraft systems within the PLF get heated up, due to the internal power dissipation of the packages and the atmospheric heating. In order to maintain the system temperatures within their acceptable levels, cool air is supplied from the umbilical tower to the inside of the PLF through an umbilical interface. The payload cooling umbilical, shown in Fig. 1, comprises a ground half and a 
vehicle half mounted on the cylindrical region of the PLF. The ground half gets separated at liftoff by the pull force exerted by a traction lanyard. The vehicle half has a shutter which closes the opening left out by the ground half separation.

The payload fairing is subjected to high external acoustics due to liftoff noise by the propulsion system, and aerodynamic noise due to turbulent pressure around the vehicle. The overall acoustic sound pressure level (OASPL) can go up to $160 \mathrm{~dB}$ [2]. This acoustic excitation of the PLF manifests as a severe vibration of the structure. Therefore, the umbilical shutter along with mechanism is qualified for these vibration inputs simulating the flight configuration as shown in Fig. 2a. The rocker clamp mechanism, shown in Fig. 2b, which has to hold the shutter in position failed to do so during the course of testing and resulted in the inadvertent opening of the shutter. This paper describes the above failure of the umbilical shutter mechanism, detailed analysis and experimentation carried out to find the cause of the failure, and the actions taken to make the system design robust.

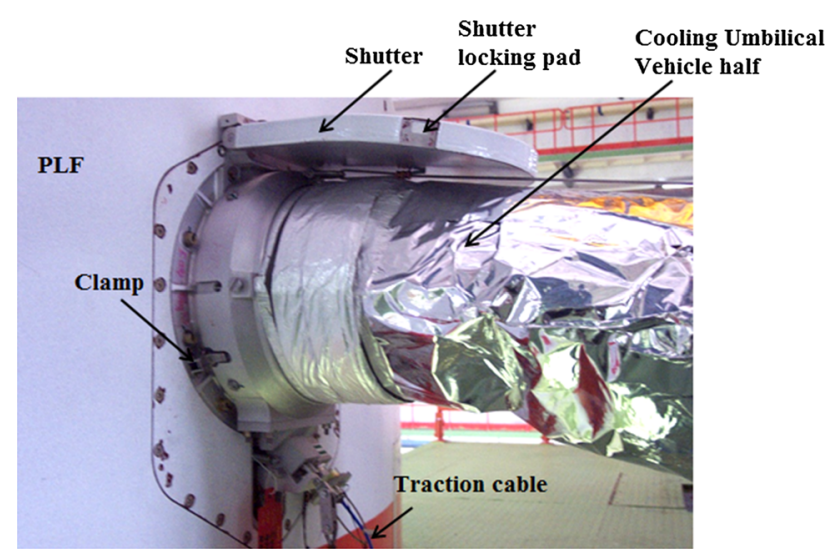

Fig. 1 Payload cooling umbilical with shutter

\section{Back Ground of Failure}

The standard practice for qualification of systems for the acoustic environment is to test for the maximum predicted acoustic level (acceptance test spectrum) with a margin of $+3 \mathrm{~dB}[1,4,5]$. The acceptance acoustic level has been finalized for the umbilical shutter enveloping the maximum predicted acoustic level at boat tail portion, which is $6 \mathrm{~dB}$ more compared to the levels at the cylindrical region of the PLF where the shutter is mounted. Though the shutter is far off from the boat tail region, this safe approach is taken partly to account for the structurally transmitted vibration and also to demonstrate the design robustness. This is giving $\mathrm{a}+6 \mathrm{~dB}$ margin in the acceptance level and $+9 \mathrm{~dB}$ margin in qualification level on the maximum predicted acoustic level at the shutter location. The acoustic test is done with the shutter in the closed condition, as shown in Fig. 2a, in the flight configuration. In the acoustic test, an OASPL close to the acceptance test spectrum only could be simulated, due to facility limitations. The random vibration test is a pragmatic test to simulate the vibration induced on the launch vehicle systems by the acoustic excitation. The test is conducted to test the ability of the components and its parts to withstand the dynamic stresses exerted by the vibration in the defined band of frequencies [3].

The realistic assessment of these vibration levels is made through dynamic characterization by subjecting the PLF to an acoustic test with vibration monitoring in the critical subsystems. The umbilical shutter performed satisfactorily during the acoustic test and was in closed condition. The vibration response was measured at critical locations during the acoustic test and was extrapolated to the specified acoustic acceptance test spectrum and enveloped to obtain the acceptance vibration test level in terms of the power spectral density (PSD) in $\mathrm{g}^{2} / \mathrm{Hz}$ as a function of frequency in $\mathrm{Hz}$. The vibration level in $\mathrm{g}_{\mathrm{rms}}$, as per standards, is enhanced by a test margin of to 1.5 times

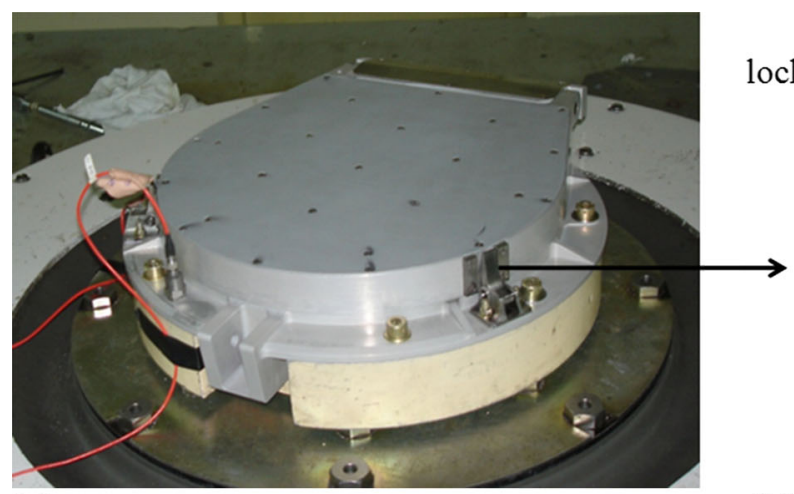

Shutter locking pad

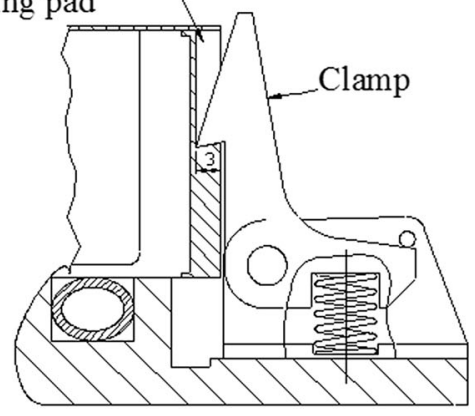

(a)

(b)

Fig. 2 Payload cooling umbilical shutter vibration test. a Vibration test configuration. b Shutter rocker clamp mechanism 
Fig. 3 Measured vibration response in the acoustic test and vibration spectrum for qualification and acceptance

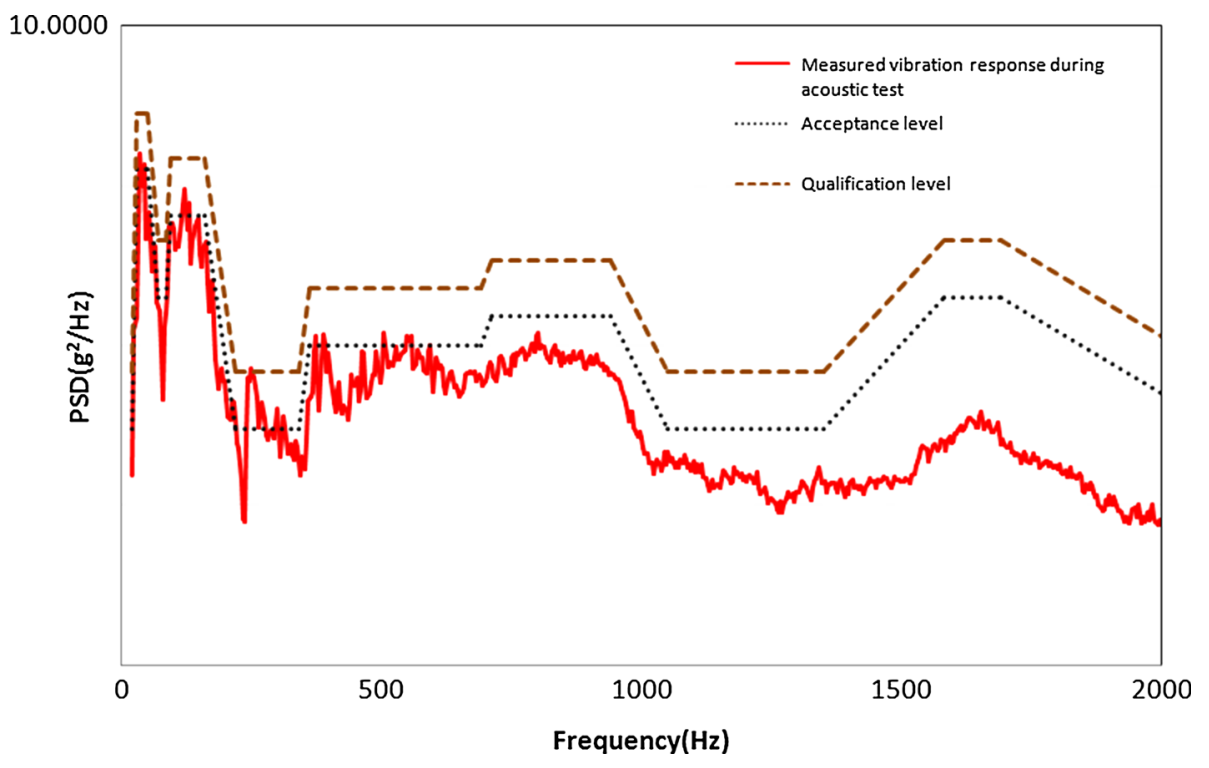

to get the qualification level $[1,4,5]$. The measured response, acceptance test level, and qualification level are given in Fig. 3.

As part of the qualification, the mechanism is tested first to the acceptance test level in a vibration table and the system withstood the vibrations satisfactorily. When the level is further enhanced to qualification level to complete the qualification testing of 2-min duration, the shutter got opened during the course of testing. The test has been aborted, and all the parts have been inspected and found in good condition.

\section{Experimentation}

The qualification test of the shutter has been carried out with vibration response monitoring on the shutter. The input PSD and the vibration response monitored on the shutter at the rocker clamp mechanism before the failure are shown in Fig. 4a. The transmissibility, computed as the square root of the ratio of the response PSD to the input PSD, is given in Fig. 4b. The transmissibility curve clearly shows that the umbilical shutter mounting is a two-degreeof-freedom system with widely spaced natural frequencies at 345 and $1200 \mathrm{~Hz}$.

\section{Failure Analysis and Numerical Results}

When analyzing the system failures during a vibration test, it is not enough to simply conclude it as due to high cycle fatigue, chatter or wear, but the analysis must relate the failure to the dynamics of the failed item and its dynamic environment [6]. When the physics of incipient failure of a system is understood unambiguously by analysis and experimentation, it can be effectively used to prevent the particular failure mode [7]. In the present analysis, dynamics of the system is used to identify the failure mode and failure mechanism, while the dynamic environment defines the failure stresses. The system functioning is studied through detailed finite element (FE) analysis, and the failure mode is identified. The frequencies of the system, the mode shapes, system damping, and the dynamic stresses on the system are assessed using the experimental data and correlated with the identified failure mode, fixing the root cause of the failure.

\section{System FE Modeling and Failure Mode Identification}

The umbilical shutter is made of an isogrid type construction, to meet the required stiffness requirements with reduced mass. It is made of aluminum alloy AA 2014 material with $20 \mathrm{~mm}$ thick. The isogrid pockets are covered by a 1-mm-thick skin sheet made of aluminum alloy 2014 and fastened to the top of the shutter at the node of the isogrid pockets. The shutter is hinged at one end and locked by two latches at the other end by a rocker clamp mechanism. The rocker clamp mechanism comprises a shutter locking pad assembled to the side face of the umbilical shutter and a spring-loaded clamp with a $10^{\circ}$ taper interface hinged on a bracket which is assembled to the shutter mounting ring at two locations, $120^{\circ}$ apart (Fig. 2a and b). The moment due to the spring force about the hinge holds the shutter in the locked condition when the shutter is closed.

The modal analysis of the shutter is carried out by importing a 3D solid model generated in solid works to the 

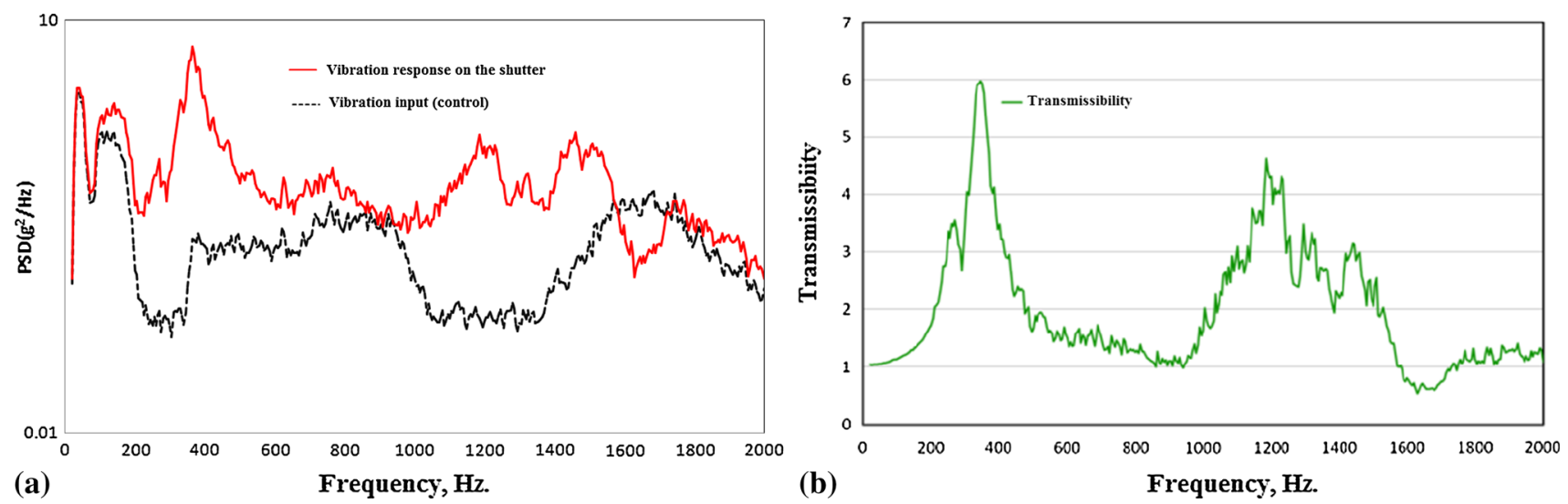

Fig. 4 Vibration response on the shutter-original design. a Vibration response on the shutter and test input spectrum. b Transmissibility curve

ANSYS $^{\circledR}$ work bench. After importing the model to ANSYS $^{\circledR}$, it is meshed with solid 186 3-D 20 node tetrahedral structural solid elements. The element size provided is $3 \mathrm{~mm}$ with a minimum edge length of $0.74241 \mathrm{~mm}$. As the shutter is having a hinge with a cylindrical rod at one end, the cylindrical support option is given for the hinge joint and the radial, axial, and tangential movements were constrained. For simulating the locking force provided by the latches on the shutter, an elastic support with a foundation stiffness of $40 \mathrm{~N} / \mathrm{mm}$ was provided at the latch locations. A modal analysis with unsymmetrical eigensolver is carried out to determine the natural frequencies and mode shape of the shutter assembly and the first mode obtained from the analysis is shown in Fig. 5.

The resonant frequency obtained for the shutter assembly from the modal analysis of Fig. 5 is $326 \mathrm{~Hz}$ as compared to $345 \mathrm{~Hz}$ observed in the test. The fundamental mode is the cantilever mode with the maximum displacement of the shutter near the shutter to clamp interface which is critical for the mechanism integrity. In this mode, the vibration forces trying to open the shutter, against the holding force exerted by the clamp, will be maximum. The forces due to random vibration cause a clockwise moment on the clamp which can result in the opening of the shutter. It is therefore essential to assess the forces and moments on the clamp and address actions for mitigating the opening of the shutter.

\section{Estimation of the Vibratory Force}

The first two modes of the system are at 345 and $1200 \mathrm{~Hz}$ as obtained from the transmissibility curve of Fig. 4b. The stress responses at higher modes are at least two orders lower compared to that at the first mode [8]. Moreover, from the first four modes which are studied, the first bending mode is giving the maximum displacement at the shutter location.

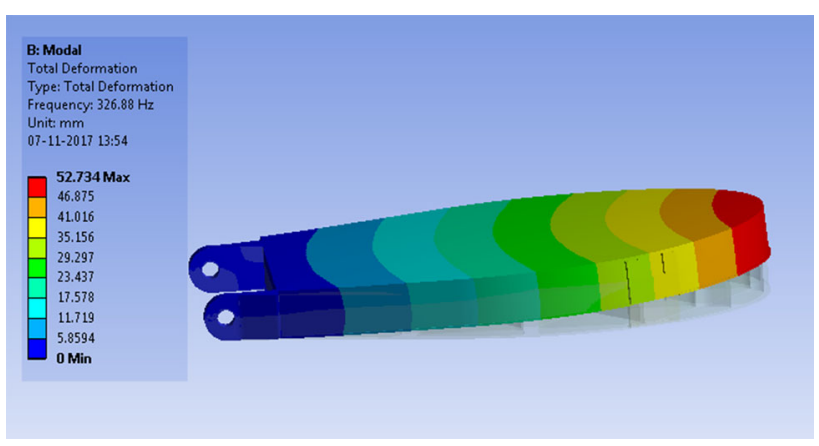

Fig. 5 First mode of the shutter assembly from the modal analysis

Considering these points, the vibration forces are estimated for the excitation at first mode with frequency $f_{\mathrm{n}}$. The half power bandwidth is defined as the bandwidth of frequency in the transmissibility curve where the transmissibility is greater than $(1 / \sqrt{ } 2)$ times the peak value, and the half power bandwidth is considered a more appropriate measure of energy dissipation in the system around the resonance [9]. Hence, to get a better picture of the energy content, around the resonance, the amplification factor at resonance ' $Q$ ' is obtained as $f_{\mathrm{n}} / \delta f_{\mathrm{n}}$, where $f_{\mathrm{n}}$ is the natural frequency and $\delta f_{\mathrm{n}}$ is the bandwidth of frequency corresponding to the half power points [9]. The ' $Q$ ' value computed using this procedure from the transmissibility curve of the shutter vibration response shown in Fig. $4 \mathrm{~b}$ is 5.75 .

The traditional method of dynamic response analysis using Miles equation is a simple and very useful for obtaining the rms acceleration response for a given input, and the response could be used to obtain the equivalent static load. The response in $g_{\text {rms }}$ is given by the following equation:

The response, $g_{\text {rms }}=\sqrt{\left\{\frac{\pi}{2}\right\} \text { fnQ }\{\text { PSD input }\}}$

where the response is root-mean-square acceleration in $g_{\text {rms }}, \quad f_{\mathrm{n}}=$ natural frequency, $\mathrm{Hz}, \quad Q=$ resonant 
amplification factor, and 'PSD input' is the input power spectral density in $\mathrm{g}^{2} / \mathrm{Hz}$ at the component natural frequency $[10,11]$. Substituting the values,

The response, $\begin{aligned} g_{\mathrm{rms}} & =\sqrt{\left\{\frac{\pi}{2}\right\} \times 345 \times 5.75 \times\{0.1275\}} \\ & =19.92 g_{\mathrm{rms}} .\end{aligned}$

The vibratory force, $F_{\mathrm{sh}}$, exerted by the shutter of mass $\mathrm{m}$ $(1.2 \mathrm{~kg})$ on the mechanism clamp is computed for the $g_{\text {peak }}$ value, which is obtained by multiplying the $g_{\text {rms }}$ value by a crest factor of 3 .

$F_{\text {sh }}=19.92 \times 3 \times 1.2 \times 9.81=70.3 \mathrm{~N}$.

As there are two mechanisms, the load per clamp is $352 \mathrm{~N}$.

\section{Margin Assessment Against Shutter Opening}

The random vibration response of the shutter with the mass of $1.2 \mathrm{~kg}$ causes the shutter locking pad to exert a force $F_{\mathrm{sh}}$ on the clamp (Fig. 1b) which results in a clockwise moment on the clamp about its hinge on the bracket. This can cause the chatter of the clamp, resulting in the opening of the shutter. The opening induced by this clockwise moment is resisted by the anticlockwise moment due to the frictional force $F_{\mathrm{f}}$ and the spring force $F_{\mathrm{s}}$. The free body diagram of the system with all the forces acting on the clamp is given in Fig. 6.

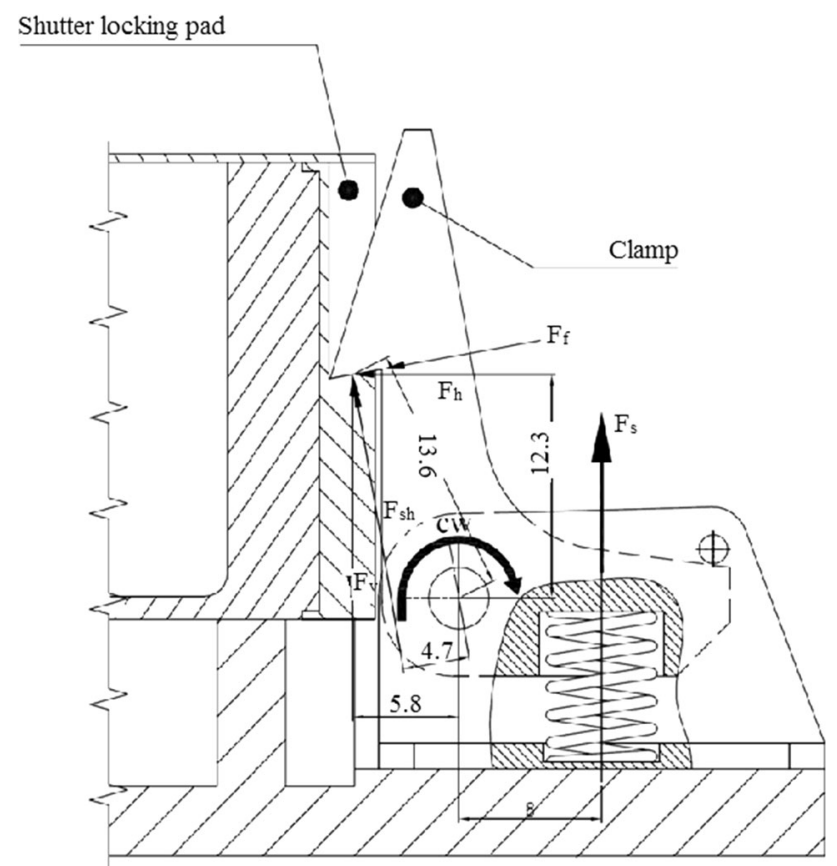

Fig. 6 Free body diagram of the rocker clamp mechanism-original design
The vibratory force of the shutter acting normal to the clamp $F_{\text {sh }}$, as computed in the preceding section, is $352 \mathrm{~N}$. Considering the clamp wedge angle of $10^{\circ}$, the vertical component of this force $F_{\mathrm{v}}$ is $346 \mathrm{~N}\left(352 \times \cos 10^{\circ}\right)$ and the horizontal component $F_{\mathrm{h}}$ is $61 \mathrm{~N}\left(352 \times \sin 10^{\circ}\right)$. The frictional force $F_{\mathrm{f}}$ at the interface with a coefficient of friction of 0.2 is $0.2 \times 352=70 \mathrm{~N}$, and the spring force $F_{\mathrm{s}}$ is $36 \mathrm{~N}$.

Taking the moments about the hinge, we get,

Clockwise moment, $\mathrm{M}_{\mathrm{CW}}=\mathrm{Fv} \times$ Moment arm

$$
=346 \times 5.8=2006.8 \mathrm{Nmm} \text {. }
$$

Anticlockwise moment due to the forces $F_{f}, F_{h}$, and $F_{s}$ and their corresponding moment arm is,

$$
\begin{aligned}
M_{\text {Acw }} & =\left(F_{\mathrm{f}} \times 13.6+F_{\mathrm{h}} \times 12.3\right)+(\mathrm{Fs} \times 8) \\
& =(70 \times 13.6+61 \times 12.3)+(36 \times 8) \\
& =1990.3 \mathrm{Nmm} .
\end{aligned}
$$

The clockwise moment on the clamp about the hinge tends to open the shutter, while the anticlockwise moment keeps the shutter closed. The analysis shows that the margin against opening is negative $\{(1990.3 / 2006.8)-1\}=-0.008$. The negative margin points toward design marginality as the cause for the mechanism to open under random vibration. The physics of failure is thus understood to be the clockwise moment tending to open the clamp exceeding the anticlockwise moment trying to keep the clamp in closed position.

\section{Design Solution and Margin Demonstration}

The mechanism was modified to reduce the clockwise moment by modifying the hinge location of the clamp and the clamp-shutter locking location in such a way that the vertical component of vibratory force passes through the hinge center, thereby reducing the clockwise moment trying to open the shutter to zero. The modified design of the mechanism and its free body diagram are shown in Fig. 7. The clockwise moment trying to open the mechanism is caused by the offset of the vertical force from the hinge center. The offset which was $5.8 \mathrm{~mm}$ in original design is zero in the modified design and by individual part tolerances is controlled to be within $0.05 \mathrm{~mm}$. The margin for this mechanism is computed conservatively assuming an offset of $1 \mathrm{~mm}$.

The vibration DQT test has been repeated with the modified rocker clamp mechanism. The vibration input and the response measured on the shutter near the mechanism is given in Fig. 8a, and its transmissibility is computed and compared with that obtained with original design in Fig. $8 \mathrm{~b}$. The response on the shutter is computed in similar 
lines of the computation made for the original design. The $Q$ value is 5.92, and the average input PSD around the half power point bandwidth is $0.0711 \mathrm{~g}^{2} / \mathrm{Hz}$.

The response $\begin{aligned} g_{\mathrm{rms}} & =\sqrt{\left\{\frac{\pi}{2}\right\} \times 320 \times 5.92 \times\{0.0711\}} \\ & =14.54 g_{\mathrm{rms}}\end{aligned}$

(Eq 6)

Force exerted by shutter on the mechanism $=14.54 \times 3 \times 1.2 \times 9.8=513 \mathrm{~N}$. The load per

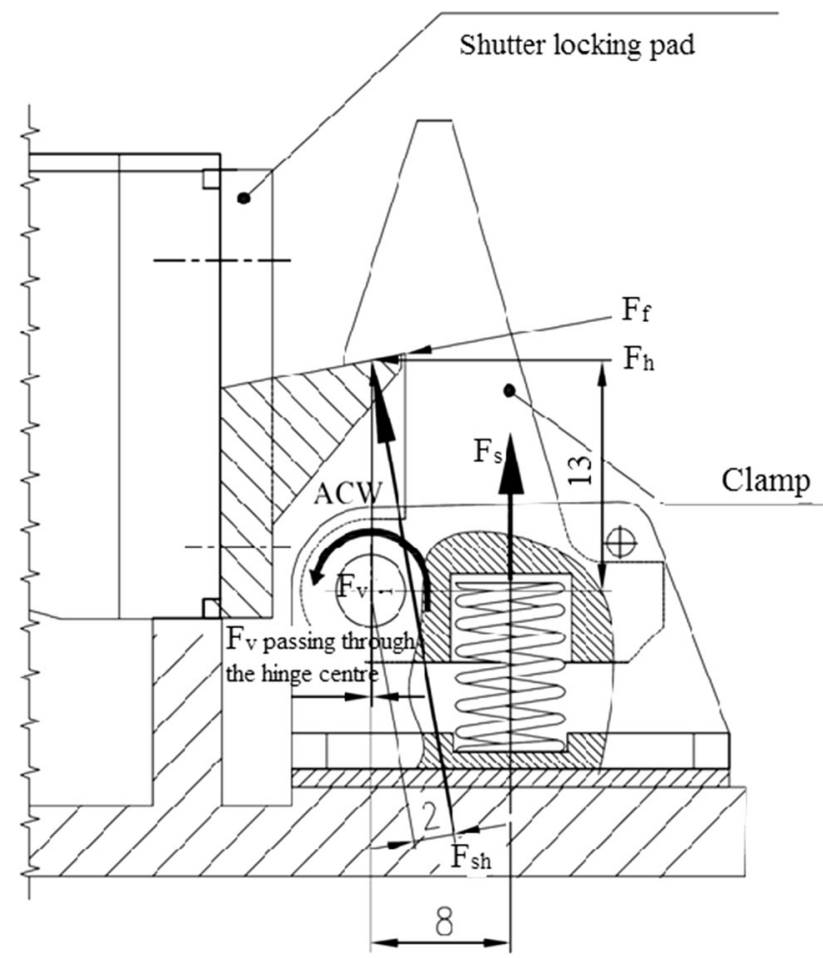

Fig. 7 Free body diagram of the rocker clamp mechanism—-modified design

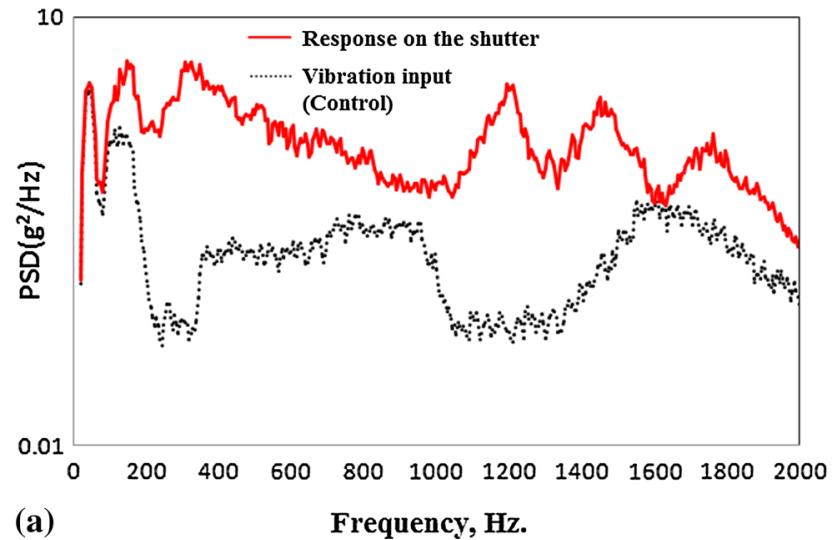

mechanism is $257 \mathrm{~N}$. Considering the clamp wedge angle of $10^{\circ}$, the vertical force $F_{\mathrm{v}}$ is $253 \mathrm{~N}$ and the horizontal force $F_{\mathrm{h}}$ is $44.62 \mathrm{~N}$. The frictional force $\mathrm{F}_{\mathrm{f}}$ at the interface with a coefficient of friction of 0.2 is $0.2 \times 257=51 \mathrm{~N}$, and the spring force $\mathrm{F}_{\mathrm{s}}$ is $36 \mathrm{~N}$. Taking the moments about the hinge, the clockwise moment is $253 \mathrm{Nmm}$ corresponding to a worst-case force line offset of $1 \mathrm{~mm}$. The anticlockwise moment trying to close the shutter is $1536 \mathrm{Nmm}$. This gives a margin of 5 against the opening of the shutter in the modified design as compared to a negative margin of 0.008 in the original design. With the modified design, the system has been further tested up to 1.5 times the qualification level to demonstrate the robustness of the system, and the system successfully withstood the vibration loads without opening of the shutter.

\section{Discussion}

The integrated shutter system along with the rocker clamp mechanism functioning and its FE model are analyzed, and the failure cause for the inadvertent opening of the mechanism is identified as the vibratory force in the first mode of the shutter causing a clockwise moment on the clamp trying to open the shutter. The dynamic behavior of the system has been assessed by the transmissibility curve as shown in Fig. 4b, which shows clearly that the system is a two-degree-of-freedom system with frequencies at 345 and $1210 \mathrm{~Hz}$. Considering that the stress responses at higher modes are at least two orders lower compared to that in the first mode and the FE analysis showing a cantilever mode with higher amplitudes at the shutter location for the first frequency, the assessment for the vibratory force is made using the $g_{\text {rms }}$ value computed from the Mile's equation, for a single-degree-of-freedom system with the first

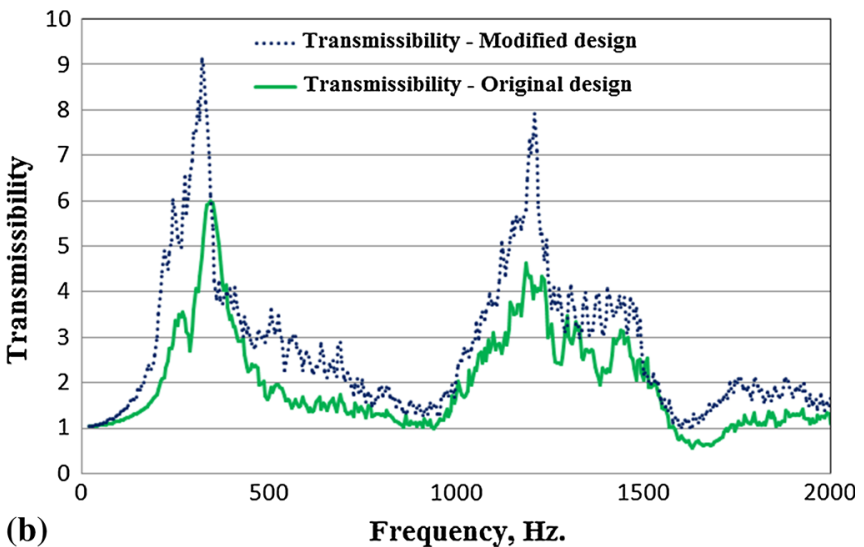

Fig. 8 Vibration response on the shutter with modified design and the transmissibility comparison with original design. a Vibration input and response on the shutter-modified design. b Transmissibility comparison with original design 
bending mode frequency of $345 \mathrm{~Hz}$. Also, the measured shutter response is $6.4 \mathrm{~g}^{2} / \mathrm{Hz}$ at $365 \mathrm{~Hz}$ which is higher as compared to $1.5 \mathrm{~g}^{2} / \mathrm{Hz}$ at $1185 \mathrm{~Hz}$. Assuming that the realized ensemble of random vibration histories is best represented by a Gaussian distribution, the $g_{\text {rms }}$ response is a $1 \sigma$ response. When a crest factor of 3 is applied to the $g_{\text {rms }}$ value, the $3 \sigma$ value is obtained which will be $99.73 \%$ of the time greater than the instantaneous random load encountered by the system. The $3 \sigma$ value, so obtained, known as peak random load factor $g_{\text {peak }}$, is used for computing vibratory forces (11). The half power point bandwidth method is used for assessing the transmissibility, and the average of PSD in this bandwidth of frequency was used for the input PSD for computation of the vibratory force.

The margin of the system against the shutter opening failure mode was obtained using the static equilibrium equations from the free body diagram of the clamp, by comparing the clockwise moment trying to open the shutter with the anticlockwise moment due to frictional and spring forces trying to keep the shutter in closed position. The margin was found to be negative 0.008 by analysis, and the problem could be compounded by the dispersions in the random vibration input, in the realized hardware dimensions and interface clearances, resulting in the inadvertent opening of the shutter. The failure mechanism is thus assessed as the vibratory forces of the shutter causing a clockwise moment on the clamp trying to open the shutter marginally exceeding the anticlockwise on the clamp.

The physics of failure has been effectively used for avoiding this failure mode in the modified design. The clamp is modified to align the vertical component of the vibratory force vector along the hinge so that the clockwise moment trying to open the shutter is avoided. The system hardware dimensions are given close tolerances to limit the distance between the vibratory force vector $F_{v}$ and hinge center within $0.05 \mathrm{~mm}$. The vibration qualification is completed satisfactorily for the modified design. From the test data, the first bending mode frequency is $320 \mathrm{~Hz}$ as compared to $345 \mathrm{~Hz}$ in the original design. The damping of $8.44 \%$ as assessed by $Q$ value is found comparable to $8.7 \%$ observed in the original design. The input PSD, from the test level, is 0.0711 at $320 \mathrm{~Hz}$ as compared to the input PSD of 0.1275 at $345 \mathrm{~Hz}$ in the original design. The resultant vibratory force is $257 \mathrm{~N}$ as compared to $352 \mathrm{~N}$ in the original design. However, the real advantage came from the alignment of the vertical component of the force along the hinge center. Even though the force vector moment arm is controlled within $0.05 \mathrm{~mm}$, the analysis is done conservatively with a moment arm of $1 \mathrm{~mm}$ and a margin of 5 was established against the opening.

Building robustness in the design is an important strategy for failure mode avoidance [7]. Hence, it is important to design systems to withstand a wide range of harsh environments and demonstrate it in the ground tests. In accordance with this concept, the vibration qualification level is finalized corresponding to $+9 \mathrm{~dB}$ of predicted acoustic spectrum at the cylindrical portion where the shutter is mounted. A positive margin of 5 is established for the DQT vibration levels based on the experimental data and theoretical analysis. The system was further tested to 1.5 times the qualification vibration levels satisfactorily, thus demonstrating the robustness of the system. Thus, the failure analysis by experimentation and analysis led to identifying the physics of failure, which was effectively used for the design modification, leading to design robustness.

\section{Conclusion}

The failure of the cooling umbilical shutter mechanism during qualification vibration test has been assessed by detailed experimentation and analysis of the vibration responses, transmissibility, and the forces and moments acting on the clamp which holds the shutter in position. Failure of the shutter mechanism is due to the vibratory force of the shutter in its first bending mode that causes a clockwise moment to act on the clamp. The clockwise moment trying to open the shutter exceeded the anticlockwise moment on the clamp due to the spring and frictional forces that keep the shutter in the closed condition. The design marginality was brought out, and the physics of failure was understood that was effectively used for the modification of the shutter clamp design. The modification brought in the design robustness and the same was demonstrated by vibration testing with huge margins.

Acknowledgments Authors wish to thank Director, VSSC, for the continuous support in carrying out the study and permitting to publish the paper. Authors gratefully acknowledge the vibration testing support and test data provided by ISDTF facility.

\section{References}

1. B.N Suresh, K. Sivan, chapter 7.9 (thermal environments) and chapter 11.11 (environmental testing for space structures), Integrated design for Space Transportation (Springer, New York, 2015), pp. 258-259, 489-491

2. Z. Ling, W. Jinbo, C. Yuanyuan, Noise control of a payload fairing through layout of acoustic blanket, in 23rd International Congress on Sound and Vibration, ICSV 23, Athens, Greece, 1014 July 2016, p 1-8

3. Method 214 A, in Random Vibration, Test method for standard Electronic and Electrical Component Parts, MIL-STD-202G (Department of Defense test method standard, USA, 2002), p 1 of 4 to 4

4. Dynamic Environmental criteria, NASA-HDBK-7005, in NASA Technical Handbook, (National Aeronautics and Space Administration, 2001), pp. 194-195 
5. A. Harper, M. Ryschkewitsch, A. Obenschain, R. Day, in General Environmental Verification Standard (GEVS) for GSFC flight programs and projects, GSFC-STD-7000, (NASA Goddard Space Flight centre, 2015), pp. 2.2-1 to 2.2-6

6. Method 527, in Multi Exciter Testing, Environmental Engineering Considerations and Laboratory test, MIL-STD-810G, (Department of Defense test method standard, USA, 2008), p $527-13$

7. D. Clausing, D.D. Frey, Improving system reliability by failuremode avoidance including four concept design strategies. Syst. Eng. 8(3), 245-261 (2005)
8. S. Ron, A. Li, Methodology for fatigue prediction of electronic components under random vibration load. Trans. ASME 123, 394-400 (2001)

9. C.W. de Silva, Vibration Damping, Control And Design (CRC Press, Taylor \& Francis group, Baco Raton, 2007), pp. 1-9-1-31

10. D.S. Steinberg, Vibration Analysis for Electronic Equipment, 3rd edn. (Wiley, Hoboken, 2010), pp. 188-216

11. H.M. Lee, A simplistic look at limit stresses from random loading, ed by G.C. Marshall (NASA Technical Memorandum108427, Space Flight Centre, 1993), pp. 1-19 